\title{
In-operando Tomography and Energy-resolved Elemental Mapping Projection X-ray Microscopy and Transmission X-ray Microscopy Beamline at TPS of NSRRC
}

\author{
Yen-Fang Song ${ }^{1 *}$, Chao-Chih Chiu ${ }^{1}$, Gung-Chian Yin ${ }^{1}$, Chek-Hai Lim² ${ }^{2}$, and Nae-Lih Wu ${ }^{2}$, \\ 1. National Synchrotron Radiation Research Center, Hsinchu 30076, Taiwan. \\ 2. Laboratory of Energy Materials, Department of Chemical Engineering, National Taiwan University, \\ 106 Taipei, Taiwan. \\ * Corresponding author, song@ nsrrc.org.tw
}

Synchrotron radiation projection X-ray microscopy (PXM) and transmission X-ray microscopy (TXM) are vital techniques for revealing directly the interior morphological micron, sub-micron and nanometer structure of the specimen. Utilizing synchrotron radiation hard X-ray with the energy range of 5 to $50 \mathrm{keV}$, PXM and TXM provide the critical ability of large probing depth up to $10 \mathrm{~mm}$ for nondestructive 3D tomography, high flux for in-situ tomography to analysis complex structure evolution, and energy tunable for energy-resolved elemental mapping. Furthermore, the aquiferous specimen is feasible because no vacuum experiment environment is required.

In order to satisfy the extensive need of PXM and TXM for many disciplines, such as energy storage, advanced materials, medical biology, environmental science, geology, and paleontology, etc., the TPS 37A PXM-TXM beamline adopting wiggler source at TPS of NSRRC is under construction for inoperando tomography and energy-resolved elemental mapping. This beamline will provide four operation modes as shown in Figure 1 (1) to (4), respectively. They are described as following: (1) White light PXM mode with the energy range of 5 to $50 \mathrm{keV}$ and $0.5 \mu \mathrm{m}$ spatial resolution. (2) Monochromatized beam PXM mode using double Mo- $\mathrm{B}_{4} \mathrm{C} / \mathrm{Si}$ multilayers with the energy range of 5 to $30 \mathrm{keV}, 1 \%$ energy resolution $(\Delta \mathrm{E} / \mathrm{E})$ and $0.5 \mu \mathrm{m}$ spatial resolution. (3) Monochromatized beam PXM mode adopting double $\mathrm{Si}(111)$ crystals with the energy range of 5 to $30 \mathrm{keV}, 1.5 \times 10^{-4}$ energy resolution $(\Delta \mathrm{E} / \mathrm{E})$ and $0.5 \mu \mathrm{m}$ spatial resolution. One vertical collimating mirror is located before double crystal monochromator (DCM) for providing the parallel beam for DCM. And one plane mirror is equipped at the downstream of DCM for controlling the beam height relative to the ground at the sample position. (4) Monochromatized beam TXM mode using double $\mathrm{Si}(111)$ crystals with energy range of 5 to $12 \mathrm{keV}$ and $1.5 \times 10^{-4}$ energy resolution $(\triangle \mathrm{E} / \mathrm{E})$. One vertical collimating mirror is located before DCM. One horizontal focus mirror and one vertical focus mirror provide the focused beam as the secondary source for the end-station of TXM. The $\mathrm{X}$-ray is refocused by a capillary condenser to the specimen and then transmits through a zone plate which provides the spatial resolution of $30 \mathrm{~nm}$. A phase ring is located at the back focal plane of the zone plate to enhance the phase contrast of the specimen which is insufficient for absorption contrast.

Here, we demonstrate a significant insight of volume expansion and phase transformation of Bi anode electrode in Na-ion batteries (NIBs) employing in-operando TXM at BL01B1 of NSRRC in Taiwan and X-ray diffraction (XRD) at BL12B1 of Spring-8 in Japan [1]. The NIBs attract the great attention and become the favorable alternation of Li-ion batteries (LIBs) for large-scale energy storage owing to the abundance and low cost of $\mathrm{Na}$. $\mathrm{Bi}$ is a promising anode material for $\mathrm{NIB}$. The $\mathrm{Na}_{3} \mathrm{Bi}$ gives the theoretical specific capacity of $385 \mathrm{mAh} / \mathrm{g}$ and the volumetric capacity density of $3750 \mathrm{mAh} / \mathrm{cm}^{3}$. Figure 2 (a) shows the TXM images of the Bi electrode particles during sodiation and (b) illustrates the corresponding voltage plot. At the end of operating the $0.57 \mathrm{~V}$ plateau, thin layers surrounding the particles were observed in panel 2, those are believed to be related with SEI formation. The shell of particles started appear from the peripheries of the particles and extended inward to form a core-shell structure (panels 3 to 5). Substantial 
volume expansion accompanied by extensive crack formation happened until the late stage of the $0.45 \mathrm{~V}$ plateau (panels 6 to 8). XRD indicated the particle expansion and fracture associated with the structural transformation from $\mathrm{NaBi}$ to $\mathrm{Na}_{3} \mathrm{Bi}$. The large particle showed sudden expansion and fracture immediately after the shell moved to the center of particle at an estimated sodiation level of $\mathrm{Na}_{2.4} \mathrm{Bi}$. Whereas, a relatively smaller particle expanded at $0.31 \mathrm{~V}$ (panel 7) corresponding to $\mathrm{Na} 2.95 \mathrm{Bi}$. And no crack was observed at the end of process with $0 \mathrm{~V}$ (panel 8). Consequently, to prevent the formation of brittle $\mathrm{Na}_{3} \mathrm{Bi}$ and reduce the particle size of $\mathrm{Bi}$ are significant for improving the cycling capacity performance. Figure 3 (a) shows the evolution of Bi particles subjected to sodiation/desodiation with limited sodiation potential to only $0.43 \mathrm{~V}$, and (b) presents the corresponding voltage profile. The particles exhibits slight and uniform expansion with about $20 \%$ diameter without cracks (panels 1 to 3 ). The dimensions of the particles remained unchanged during the following desodiation (panel 4). This result indicates the delay of the transformation of $\mathrm{NaBi}$ to $\mathrm{Na}_{3} \mathrm{Bi}$ may provide an opportunity for the $\mathrm{Bi}$ particulate anode to perform high capacity and cycling by avoiding the particle fracture or pulverization. The consequences suggest a new direction for engineering fracture-free alloying anode materials for NIBs and LIBs applications.

(1)

(3)
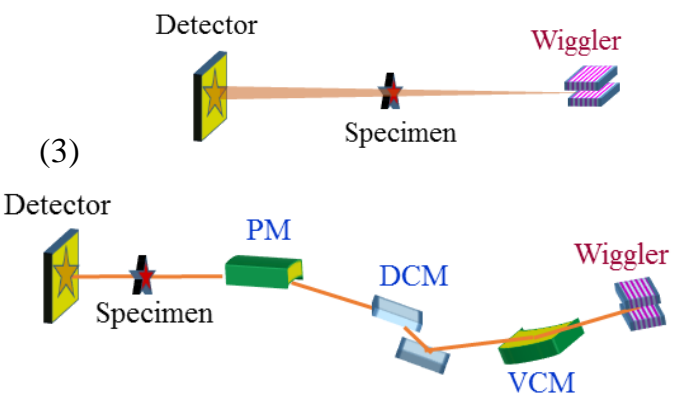

(2)

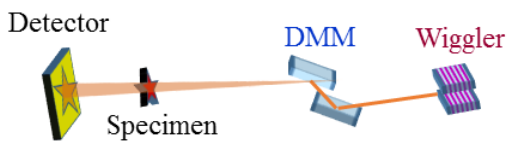

(4)

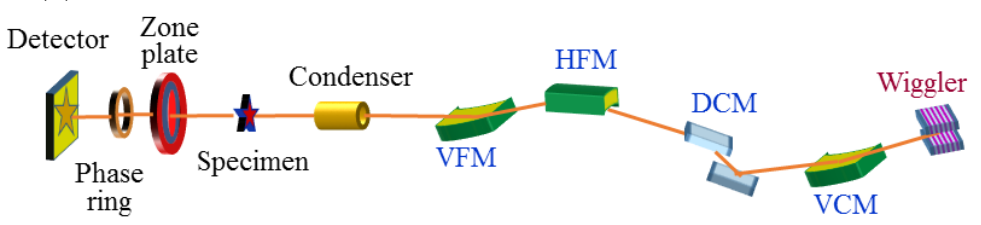

Figure 1. Layout of TPS 37A PXM-TXM beamline with four operation modes.

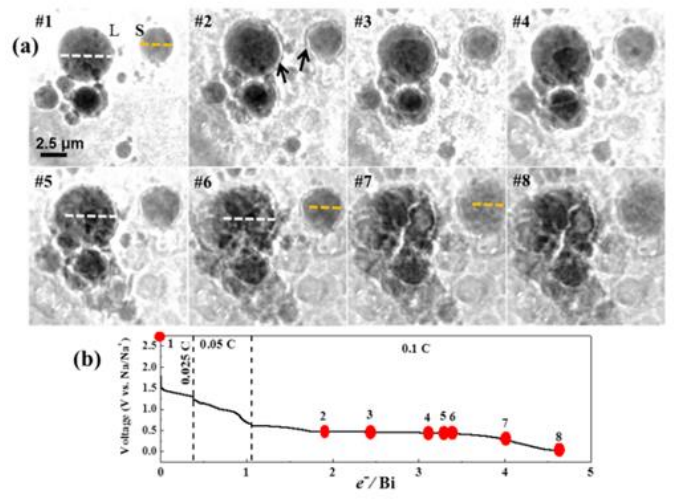

Figure 2. TXM images of Bi electrode particles during sodiation (a) with corresponding moments in the voltage plot (b). Reproduced from Ref. 1 with permission from the Royal Society of Chemistry.

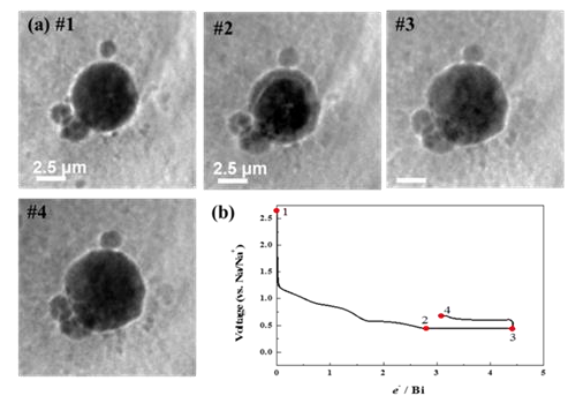

Figure 3. TXM images of Bi particles through sodiation/desodiation with limited sodiation potential (a) with corresponding moments in the voltage profile (b). Reproduced from Ref. 1 with permission from the Royal Society of Chemistry.

\section{Reference}

1. C. H Lim, B. Selvaraj, Y. F. Song, C. C. Wang, J. T. Jin, S. S. Huang, C. H. Chuang, H. S. Sheu, Y. F. Liao, and N. L. Wu, "Insight into microstructural and phase transformation in electrochemical sodiationdesodiation of bismuth particulate anode", J. Mater. Chem. A 5, 21536-21541 (2017). 